\title{
Effects of alcohol on simultaneous incentive contrast
}

\author{
W. MILES COX \\ North Chicago Veterans Administration Medical Center, Chicago, Illinois \\ and University of Health Sciences/The Chicago Medical School, Chicago, Illinois
}

\begin{abstract}
The effects of alcohol on female albino rats' runway performance were studied in a simultaneous incentive contrast effects experiment involving shifts in magnitude of food reward. Fluiddeprived animals drank an alcoholic fluid (a $10 \%$ sucrose solution containing $4.8 \%$ ethanol) or a nonalcoholic fluid (a $10 \%$ sucrose solution) immediately prior to the conditioning trials. Differential responding for large and small reward was less pronounced among the animals consuming the alcoholic fluid than among those consuming the nonalcoholic fluid. However, incentive contrast effects were not clearly demonstrated under either fluid condition. Thus, contrary to expectations, alcohol did not appear to affect emotional reactions to shifts in reward.
\end{abstract}

Since Bower's (1961) initial experiment with differential conditioning using different magnitudes of food reward, simultaneous incentive contrast effects have been extensively studied with animals in the runway (Black, 1976; Cox, 1975; Flaherty, 1982). With the simultaneous paradigm, animals are trained on intermixed trials to traverse two distinctly different runways, usually a black one and a white one. Experimental animals receive a small reward in one runway and a large reward in the other. Performance of the experimental group in its large-reward runway is compared with that of a control group that receives the large reward in both runways (test for positive contrast), and performance of the experimental group in its small-reward runway is compared with that of a control group that receives the small reward in both runways (test for negative contrast). Experimental animals respond more slowly for the small reward than control animals that receive only the small reward. Conversely, experimental animals do not respond more rapidly for the large reward than control animals that receive only the large reward, unless special training procedures are instituted (e.g., Cox, 1981). Explanations for the failure to obtain positive contrast have been discussed previously (see Black, 1976; Cox, 1975).

It has been suggested that animals' emotional reactions to shifts in rewards underlie incentive contrast effects. Thus, animals receiving an upshift in reward have been described as "elated" (Crespi, 1942), and animals receiving a downshift in reward have been described as "depressed" (Crespi, 1942) or "frustrated" (Amsel, 1962). ${ }^{1}$ Alcohol (as well as other psychoactive drugs) would be expected to alter the observed incentive contrast effects if they are, in fact, mediated by emotional reactions to shifts in rewards. Thus, if alcohol reduces negative emotional reactions, it may attenuate or eliminate the

Requests for reprints should be sent to W. Miles Cox, Psychology Service (116B), North Chicago VA Medical Center, 3001 Green Bay Road, North Chicago, IL 60064. negative incentive contrast effect. If alcohol enhances positive emotional reactions, it may produce a positive incentive contrast effect where none ordinarily occurs. ${ }^{2}$ In the present experiment, I tested these possibilities using the standard design for simultaneous incentive contrast effects. In a previous experiment (Cox, 1988), I tested the effects of alcohol on successive incentive contrast effects.

\section{METHOD}

\section{Subjects}

The subjects were 60 female albino rats (Holtzman, Madison, WI) that were approximately 90 days old at the beginning of training.

\section{Apparatus}

Two straight runways were used, one of which was painted flat black and the other of which was painted flat white. Otherwise, they were identical to the runway used by Cox (1988).

\section{Design}

Six groups, consisting of 10 animals each, were run. Three groups drank an alcoholic fluid prior to their conditioning trials, and three groups drank a nonalcoholic fluid. Within each fluid condition, one experimental group and two control groups were run. One control group received the large reward in both the black and the white runways, and the other control group received the small reward in both runways. Half of the experimental group received the large reward in the black runway and the small reward in the white runway, and the other half received the large reward in the white runway and the small reward in the black runway. The six groups provided a test for simultaneous positive contrast and simultaneous negative contrast under both alcoholic and nonalcoholic fluid conditions.

\section{Procedure}

Prior to the experiment, the animals were placed on food deprivation that maintained their body weights at $85 \%$ of their ad-lib values and fluid deprivation that allowed them $15 \mathrm{~min}$ of daily fluid exposure. The purpose of fluid deprivation was to ensure the animals' consumption of the fluids that were administered during experimental testing. The nonalcoholic fluid was a $10 \%$ sucrose solution, and the $4.8 \%$ ethanol solution contained $5 \mathrm{ml}$ of $95 \%$ ethanol per $95 \mathrm{ml}$ of the $10 \%$ sucrose solution.

Each animal was exposed to the appropriate fluid in its home cage for $\mathbf{1 5} \mathrm{min}$ immediately prior to the conditioning trials in the runway. Each trial was rewarded with a small or large food reward (1 or 10 45mg Noyes food pellets). Each day, all animals received two trials in the black runway and two trials in the white runway. The order of the trials in the two runways was determined by a recursive BWWB WBBW 
order. This procedure continued for $\mathbf{2 8}$ days, after which four daily extinction trials were given for 6 days. During the extinction phase, the animals drank the same fluid as during the training phase, but they received no food in the goalbox.

\section{RESULTS}

\section{Body Weights}

All groups of animals gained in body weights during the course of training $[F(27,1458)=2.48, p<.001]$. However, there were no group differences in body weights $(F<1)$. The mean weight of all groups was $212.7 \mathrm{~g}$.

\section{Fluid Consumption}

The animals showed gradual increases in the volume of fluid consumed during training $[F(27,1566)=35.77$, $p<.001$ ], and the groups that drank the nonalcoholic fluid drank more (daily mean consumption $=21.6 \mathrm{ml}$ ) than groups that drank the alcoholic fluid (daily mean consumption $=14.9 \mathrm{ml})[F(1,58)=132.76, p<.001]$.

\section{Runway Performance}

Latencies were first converted into speeds $(\mathrm{cm} / \mathrm{sec})$. In order to follow the customary procedure of having data points that consist of the mean of at least four trials, for each animal in the experimental groups, the two trials in each runway on each odd-numbered day were averaged with the two trials in that same runway on the following even-numbered day. Similarly, the four trials of each animal in each of the two control groups on each oddnumbered day were averaged with that animal's trials on the following even-numbered day. All subsequent analyses were based on these averages. Run and goal speeds are similar, but group differences are more pronounced for run speeds, and only they are presented here.

Run speeds of the three groups consuming the nonalcoholic fluid are shown in Figure 1, which indicates pronounced improvements in performance early in training, emerging differences among groups and conditions, and stabilized speeds and some convergence of groups and conditions later in training. The differences among groups and conditions reflect appropriate responding to the magnitude of the reward received in the runway. For example, the large-reward control group ran more rapidly than the small-reward control group $[F(1,18)=4.52$, $p<.05$ ], and the experimental group ran more rapidly for large reward than for small reward $[F(1,9)=5.98$, $p<.04]$. The departure in run speeds of the experimental group for large and small rewards occurred early in training and persisted during most of the experiment. Moreover, by Trial Block 4 the experimental group was running more slowly for small reward than the control group that received only the small reward, and this negative contrast effect graphically became more pronounced as training progressed. However, statistical analysis of the run speeds of these two groups yielded a significant group $\times$ trial blocks interaction $[F(13,234)=2.06$, $p<.02$ ], but not a significant effect for groups $(F<1)$. Separate analysis of Trial Blocks 5-9 (where graphically the negative contrast effect is most pronounced) revealed neither an effect for groups $[F(1,18)=1.30, p<.27]$ nor an interaction between groups and trial blocks $(F<$ 1). On the other hand, there was not even graphical evidence for a positive contrast effect. By Trial Block 6, the run speeds of the large-reward experimental group had

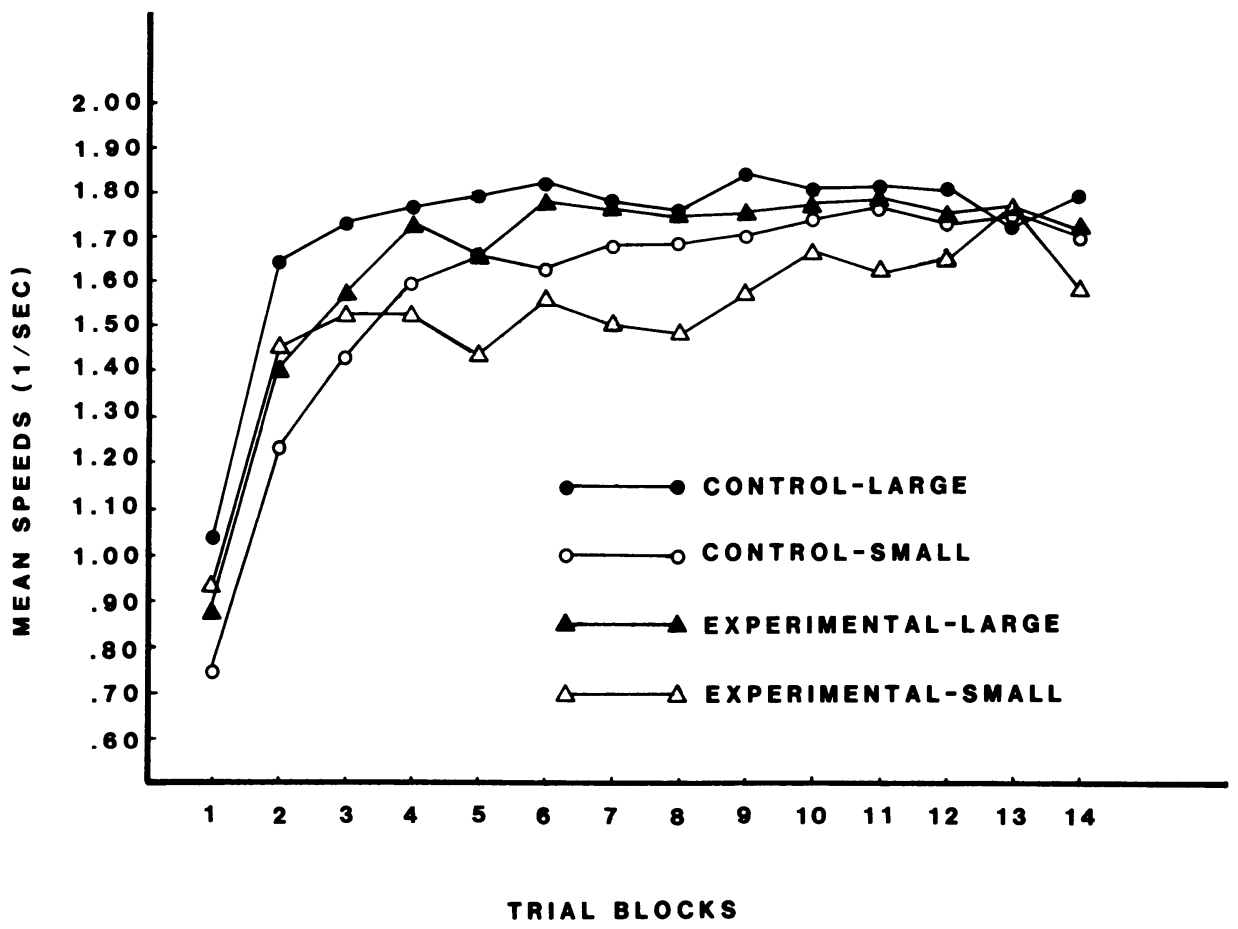

Figure 1. Run speeds during the acquisition phase of the three groups that consumed the nonalcoholic nuid. 
approximated those of the large-reward control group. Thereafter, these two groups ran at comparable speeds, with the speeds of the former group never exceeding those of the latter. Analysis revealed neither an effect for groups $[F(1,18)=1.25, p<.28]$ nor a groups $\times$ trial blocks interaction $[F(13,234)=1.51, p<.11]$.

The performance of the three groups consuming the alcoholic fluid is shown in Figure 2, which indicates increases in speeds early in training and asymptotic speeds later in training. Some of the groups and conditions diverged early in training and then converged later in training, whereas other groups and conditions first diverged later in training. In general, however, the performance of the alcoholic groups was less meaningfully related to reward magnitude than we observed in Figure 1 for the animals consuming the nonalcoholic fluid. For example, although graphically on Trial Blocks 9-14 the control group receiving a large reward ran more rapidly than the control group receiving a small reward, this difference was not statistically significant $(F<1)$. On the other hand, the experimental group did run more rapidly for a large reward than for a small reward $[F(1,9)=9.14, p<.01]$. During the first part of training (i.e., on Trial Blocks 1-6), the run speeds of the experimental group receiving a small reward were clearly depressed below those of the control group receiving a small reward $[F(1,18)=4.36$, $p<.05]$. However, it is questionable whether this should be regarded as a "real" negative contrast effect, due to (1) the lack of differential responding by the control groups to the large and small rewards, and (2) the fact that the differential conditioning animals ran slower than the small-reward control group on both large and small reward trials, and this difference was present from the very first trial block of training. Moreover, during the latter part of training (i.e., on Trial Blocks 7-14), the experimental and control groups did not respond differently to the small reward $(F<1)$. There was also no evidence of a positive contrast effect. In fact, during the first part of training (i.e., on Trial Blocks 1-6), the run speeds of the experimental group receiving a large reward were depressed below those of the control group receiving a small reward $[F(1,18)=5.03, p<.04]$. During the latter part of training (i.e., on Trial Blocks 7-14), the run speeds of these groups did not differ $(F<1)$.

The run speeds of the nonalcoholic and alcoholic groups during the extinction phase are shown in Figures 3 and 4. Extinction of the nonalcoholic groups appears more precipitous than that of the alcoholic groups, but clear group differences are not apparent within either fluid condition. Two analyses were performed to assess the effects of fluid condition on performance during this phase. An analysis that included the four control groups and the performance of the two experimental groups in the runway in which they had received small rewards during Phase 2 indicated a significant interaction of fluid condition and trial blocks $[F(2,116)=3.40, p<.04]$, but no main effect for fluid $(F<1)$. The interaction reflects, as noted above, more rapid decline in speeds by the nonalcoholic than the alcoholic groups. The second analysis that included the four control groups and the performance of the two experimental groups in the runway in which they had received large rewards during Phase 2 indicated neither a significant effect for fluid $(F<1)$ nor a significant interaction between fluid and trial blocks $[F(2,116)=2.15, p<.12]$.

\section{DISCUSSION}

In the present experiment, incentive contrast effects were not clearly demonstrated under either the alcoholic or the nonalcoholic fluid conditions. Thus, the present results provide no clear evidence that alcohol altered the animals' emotional reactions to upshifts or downshifts in reward. Nevertheless, consuming the alcoholic fluid prior to the conditioning trials interfered with the animals' differential responses to the large and small rewards.

There are several possible reasons for the failure to obtain incentive contrast effects in this experiment. The first reason is related to the man-

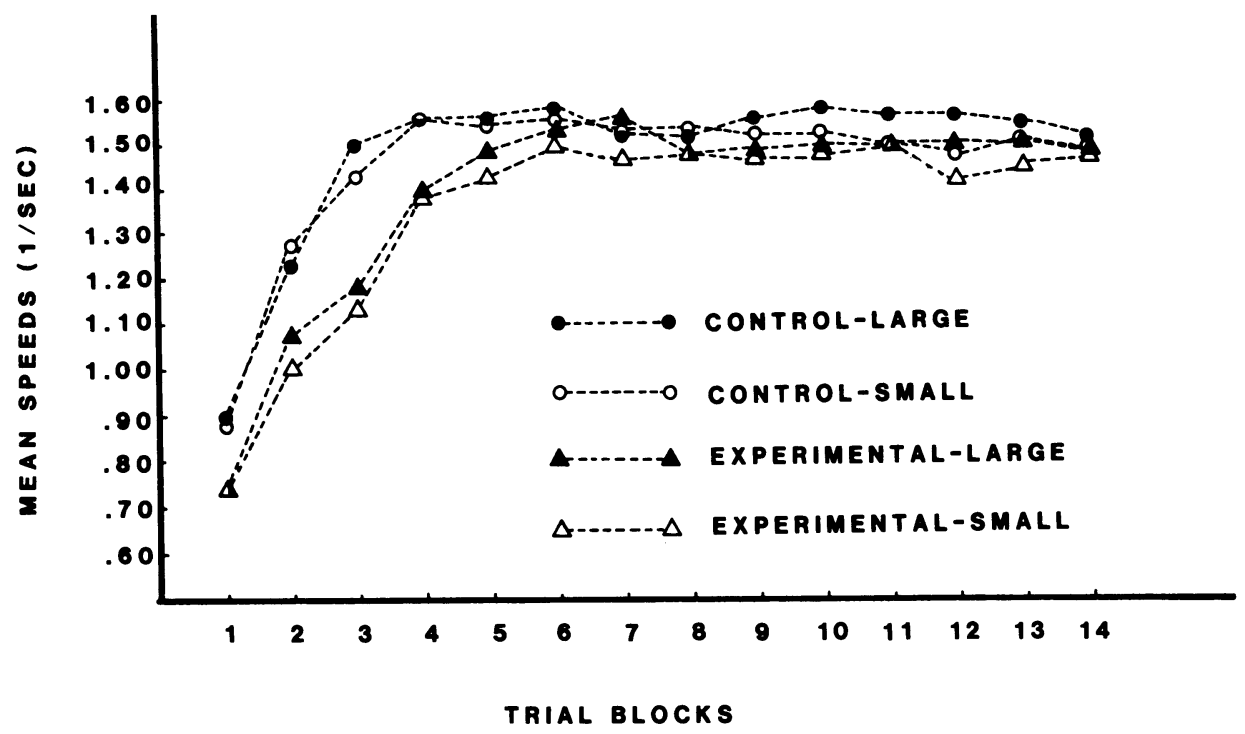

Figure 2. Run speeds during the acquisition phase of the three groups that consumed the alcoholic fluid. 


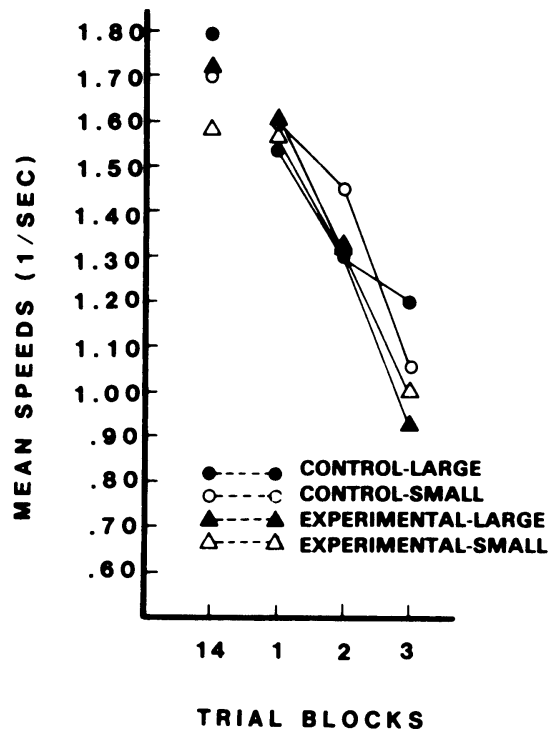

Figure 3. Run speeds during the extinction phase of the three groups that consumed the nonalcoholic fluid.

ner in which the alcoholic/nonalooholic fluids were administered. Allowing the animals to consume the fluids voluntarily avoided the aversive aspects of other methods of administration, such as intravenous injections, but it deprived the experimenter of precise control over the dose of alcoholic or nonalcoholic fluids that the animals received. Since the animals consuming the nonalcoholic fluid drank more fluid than those consuming the alcoholic fluid, the former animals presumably were less hungry than the latter animals during the conditioning trials. Moreover, since both the alcoholic and the nonalcoholic fluids were high in caloric value, consuming these fluids served to suppress the hunger of both groups of animals.

Another possible reason for the negative outcome of the present study is that the animals explored the runway prior to the conditioning trials, and "preexploration" has been found to decrease the magnitude of incentive contrast effects (Weinstock, 1971). Additionally, in future studies, start speeds, which are strongly affected by incentive manipulations, should be measured separately from run speeds. Finally, since the magnitude of both incentive effects and alcohol effects may decrease across successive trials, individual trials could be analyzed separately from trial blocks in future studies.

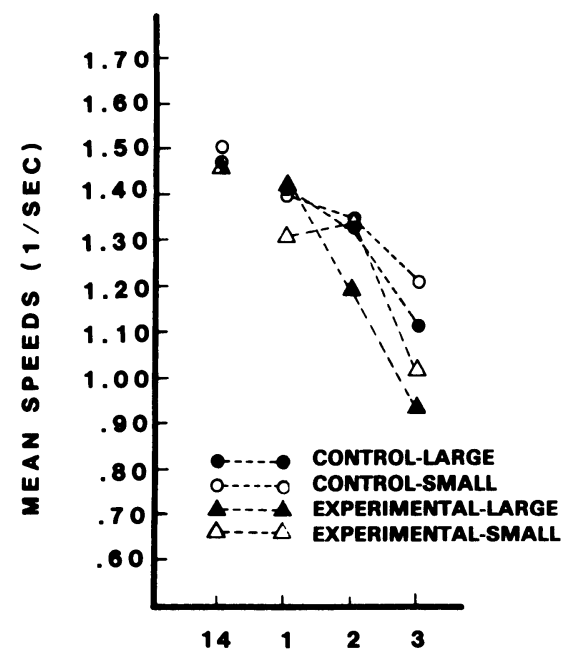

TRIAL BLOCKS

Figure 4. Run speeds during the extinction phase of the three groups that consumed the alcoholic fluid.
In spite of these apparent procedural difficulties in the present experiment, in other studies using similar procedures (e.g., Cox, 1981, 1988), incentive contrast effects were clearly obtained and the magnitude of these effects was clearly different among animals consuming the alcoholic and nonalcoholic fluids. In those studies, alcohol altered animals' reaction to their food rewards, presumably by changing their emotional reactions to them.

\section{REFERENCES}

AmSEL, A. (1962). Frustrative nonreward in partial reinforcement and discrimination learning: Some recent history and a theoretical extension. Psychological Review, 69, 306-328.

BEVAN, W. (1966). An adaptation-level interpretation of reinforcement. Perceptual \& Motor Skills, 23, 511-531.

BLACK, R. W. (1968). Shifts in magnitude of reward and contrast effects in instrumental and selective learning: A reinterpretation. Psychological Review, 75, 114-126.

BLACK, R. W. (1976). Reward variables in instrumental conditioning: A theory. In G. H. Bower (Ed.), The psychology of learning and motivation (Vol. 10, pp. 199-244). New York: Academic Press.

BowER, G. H. (1961). A contrast effect in differential conditioning. Joumal of Experimental Psychology, 62, 196-199.

CAPALDI, E. J. (1967). A sequential hypothesis of instrumental learning. In K. W. Spence \& J. T. Spence (Eds.), The psychology of learning and motivation (Vol. 1). New York: Academic Press.

Coluer, G., Mark, M. H. (1959). Changes in performance as a function of shifts in the magnitude of reinforcement. Journal of Experimental Psychology, 57, 305-309.

Cox, W. M. (1975). A review of recent incentive contrast studies involving discrete trial procedures. Psychological Record, 25, 373-393.

Cox, W. M. (1981). Simultaneous incentive contrast effects with alcoholic and nonalcoholic beverages as the discriminanda for reward magnitude. Physiological Psychology, 9, 276-280.

Cox, W. M. (1988). Effects of alcohol on successive incentive contrast. Bulletin of the Psychonomic Society, 26, 67-70.

CRESPI, L. P. (1942). Quantitative variations of incentive and performance in the white rat. American Journal of Psychology, 55, 467-517.

FlaherTy, C. F. (1982). Incentive contrast: A review of behavioral changes following shifts in reward. Animal Learning \& Behavior, 10, 409-440.

HeLsoN, H. (1966). Some problems in motivation from the point of view of the theory of adaptation level. In D. Levine (Ed.), Nebraska symposium on motivation. Lincoln: University of Nebraska Press.

McHose, J. H. (1970). Relative reinforcement effects: $S_{1} / S_{1}$ and $S_{1} / S_{2}$ paradigms in instrumental conditioning. Psychological Review, 77, 135-146.

RABIN, J. S. (1975). Effects of varying sucrose reinforcers and amobarbital sodium on positive contrast in rats. Animal Learning \& Behavior, 3, $290-294$.

Weinstock, R. B. (1971). Preacquisition exploration of the runway in the determination of contrast effects in the rat. Journal of Comparative \& Physiological Psychology, 75, 107-115.

\section{NOTES}

1. Other theoretical explanations of incentive contrast effects include perceptual ones (Bevan, 1966; Collier \& Marx, 1959; Helson, 1966), which hold that animals respond to reward according to their perceived magnitude, determined by the context in which the rewards are received. Perceptual theories, like emotional ones, predict both positive and negative contrast. Associative theories (Black, 1968, 1976; Capaldi, 1967; McHose, 1970), on the other hand, predict that with ordinary training procedures, negative contrast, but not positive contrast, will occur, and contrast effects are explained in terms of animals' responses to the actual physical properties of rewards.

2. Prior studies of the effects of psychoactive drugs other than alcohol on incentive contrast effects, summarized by Cox (1975, p. 379), have found that CNS depressants sometimes eliminate negative contrast but do not affect positive contrast. Additional findings related to drug and lesion effects on incentive contrast are discussed by Rabin (1975) and Flaherty (1982).

(Manuscript received for publication November 10, 1987.) 